age of 45 years at the time of diagnosis. Data were abstracted regarding age, parity, diabetes, hypertension, poly cystic ovaries, body mass index (BMI), tumor histology, grade, stage, and survival. Clinical and pathological characteristic were compared, and statistical analyses were performed using SPSS version 22.0.

Results: The mean age at the time of diagnosis was 38.50 years (range, 34.50-41.25) and mean BMI $\left(\mathrm{kg} / \mathrm{m}^{2}\right)$ was 30.55 (range, 27.23-38.45). 50\% patients were obese (BMI >30 kg/m²) and $40 \%$ were overweight (BMI $25-30 \mathrm{~kg} / \mathrm{m}^{2}$ ). Only 5 out 10 women had nulliparity, however, $70 \%$ women had history of polycystic ovaries, confirmed with ultrasound or on histopathological specimen. Family history was also found to be strongly associated with endometrial cancer with $70 \%$ prevalence rate. The prevalence of diabetes mellitus, hypertension and hypothyroidism were $20 \%, 10 \%$ and $10 \%$ respectively. Seven patients (70\%) had well differentiated tumors, 2 (20\%) moderately differentiated and $1(10 \%)$ had poorly differentiated tumor. Stage $1 \mathrm{~A}$ disease was found in $7(70 \%)$ patients, stage $1 \mathrm{~B}$ in $1(10 \%)$, stage 2 in $1(10 \%)$ and stage 3 in $1(10 \%)$ patient. $20 \%$ patients had completed 5 -years disease free interval, one patient was expired with recurrence and stage 3 disease, while 1 woman was lost to follow up after surgery.

Conclusion: We conclude that the obesity, family history and polycystic ovaries are strongly associated risk factors for endometrial cancer in women aged 45 years or younger. We could not find any significant association with medical disorders such as diabetes and hypertension. Nulliparity seems to have less strong relationship with development of endometrial cancer. Majority of young patients have early-stage disease with well differentiated tumors and favorable histology.

Poster (E15)

Endometrial Hyperplasia, Endometrial Intra-epithelial Neoplasia, and Endometrial Cancer

https://doi.org/10.3802/jgo.2021.32.S1.E15

\section{Survival benefit of para-aortic lymph node evaluation in endometrioid endometrial carcinoma}

\section{Khemanat Khemworapong, ${ }^{1, *}$ Pattra Wisarnsirirak, ${ }^{2}$ Atthapon Jaishuen, 'Boonlert Viriyapak,, Pisutt Srichaikul, ${ }^{1}$ Vuthinun Achariyapota, ${ }^{1}$ Nida Jareemit ${ }^{1}$}

'Mahidol University, Nakhon Pathom, Thailand

(khemanatkhem@gmail.com)

${ }^{2}$ Thammasat University, Bangkok, Thailand

Objective: To evaluate the 5-year survival in endometrioid endometrial carcinoma patients, who underwent retroperitoneal pelvic lymph nodes (PLN) evaluation alone, compared to both pelvic and para-aortic lymph nodes (PPALN) evaluation.

Methods: This retrospective cohort study enrolled 654 women who were diagnosed with endometrioid endometrial carcinoma and underwent surgical staging at Siriraj Hospital, Mahidol University between January 2006 and December 2015. Patients with PLN evaluation ( $\mathrm{n}=266)$ and patients with PPALN evaluation $(n=388)$ were included. We excluded patients with no retroperitoneal lymph node evaluation, history of any cancer within 5 years, previously received chemotherapy or radiotherapy. All results were recorded and statistically analyzed.

Results: Mean follow-up time was 83.0 months. The 5-year overall survival (OS) in PLN and PPALN groups were 56.72 months (95\% confidence interval $[\mathrm{CI}]=55.37-58.07)$ and 57.52 months (95\% CI=56.57-58.47), respectively ( $p=0.096)$. The 5-year disease specific survival (DSS) in PLN group were 57.15 months $(95 \% \mathrm{CI}=55.84-58.45)$ in PLN group and 59.03 months (95\% CI=58.44-59.61) in PPALN group, respectively ( $\mathrm{p}=0.027$ ). Conclusion: The OS was not significantly difference between patients who underwent PPALN evaluation compared to patients who underwent PLN alone, although there was a trend to improve OS for patients with PPLAN. DSS was significantly better for patients who received PPALN evaluation compared to patients with PLN alone.

Poster (E16)

Endometrial Hyperplasia, Endometrial Intra-epithelial Neoplasia, and Endometrial Cancer

https://doi.org/10.3802/jgo.2021.32.S1.E16

\section{Prevalence of occult endometrial carcinoma in patients with endometrial intraepithelial neoplasia underwent hysterectomy at Siriraj Hospital}

\author{
Waraphon Thongsang, ' Sompop Kuljarusnont, \\ Suchanan Hanamornroongruang \\ Siriraj Hospital, Bangkok, Thailand (pimpimwaraphon@gmail.com)
}

Objective: To determine the prevalence and pre-operative risk factors of occult endometrial carcinoma in the patients with endometrial intraepithelial neoplasia (EIN) who underwent hysterectomy at Siriraj Hospital.

Methods: One hundred and sixty EIN patients diagnosed between January 2007 and December 2020 were enrolled. Patients who did not undergo surgery as a primary treatment were excluded. All pathological slides were reviewed by one 
experienced gynecologic pathologist. Data were retrieved from the medical records, including gynecologic data, sonographic findings, surgical and pathological results. The prevalence of endometrial carcinoma in such patients was calculated. Various characteristics were used to determine the associated risk factors. Results: Ninety-eight patients were analyzed. Twenty-nine patients (29.6\%) were subsequently diagnosed with stage I endometrial carcinoma after hysterectomy. The hysterectomy pathologies of 69 patients (70.4\%) were EIN and other benign diagnoses. The majority of patients who diagnosed with endometrial carcinoma were pre-menopause $(80 \%, \mathrm{p}=0.458)$ and nulliparous $(70 \%, \mathrm{p}=0.045)$. Endometrial thickness greater than $2 \mathrm{~cm}$ and body mass index (BMI) greater than $30 \mathrm{~kg} / \mathrm{m}^{2}$ were demonstrated as two associated risk factors for occult endometrial carcinoma with the odds ratio of 9.7 and 2.5, respectively.

Conclusion: Occult endometrial carcinoma was observed in approximately one-third of the patients with pre-operative EIN diagnosis. Endometrial thickness greater than $2 \mathrm{~cm}$ and BMI greater than $30 \mathrm{~kg} / \mathrm{m}^{2}$ were associated risk factors for occult endometrial carcinoma.

Poster (E17)

Endometrial Hyperplasia, Endometrial Intra-epithelial Neoplasia, and Endometrial Cancer

https://doi.org/10.3802/jgo.2021.32.S1.E17

\section{A window of opportunity study of nivolumab in early-stage endometrial cancer patients with MMRd who had failure after progestins for fertility-sparing treatment (NIVEC)}

Young Hwa Kwak, Junsik Park, Yun-Jung Go, Sunghoon Kim, Jung-Yun Lee*

Yonsei University College of Medicine, Seoul, Korea (JUNGYUNLEE@yuhs.ac)

Background: National Comprehensive Cancer Network guidelines recommend high-dose progesterone for fertilitysparing treatment only for patients with clinical stage IA, grade 1 without myometrial invasion. In patients with treatment failure from progestins, there is no treatment option except hysterectomy, even if the patients want to preserve the uterus. Recently, some studies evaluated the role of prognostic significance of the Proactive Molecular Risk Classifier for endometrial carcinoma classification infertility-sparing management of endometrial cancer. They showed that patients with mismatch repair deficiency (MMRd) had a lower response rate than those with mismatch repair proficiency with hormone therapy. MMRd tumors are known to be highly immunogenic and of great interest for immune checkpoint inhibitor. Results from a phase I/II study (CA209003/MDX1106-03) indicate that nivolumab (BMS-936558; anti-PD-1 monoclonal antibody) is active in multiple tumor types. In this regard, we suggest a single-arm phase II study investing the efficacy of nivolumab in the fertility-sparing management of endometrial cancer patients with MMRd who had failure with progestins.

Methods: Early-stage endometrial cancer patients with MMRd who had failure after progestins for fertility-sparing treatment and desire to preserve fertility was offered nivolumab. Approximately 8 patients will be included from 6 centers in Korea for 3 years. The primary endpoint of the study is to determine the efficacy of nivolumab by assessment of complete response.

Poster (E18)

Endometrial Hyperplasia, Endometrial Intra-epithelial Neoplasia, and Endometrial Cancer

https://doi.org/10.3802/jgo.2021.32.S1.E18

\section{Prognostic factor of pretreatment thrombocytosis to endometrial cancer in Bhumibol Adulyadej Hospital}

\section{Arun Jevae, ${ }^{1}$ Piyawan Pariyawateekul,, ${ }^{1, *}$ Worrawan Sirichai, ${ }^{1}$ Kornkarn Bhamarapravatana, ${ }^{2}$ Komsun Suwannarurk ${ }^{3}$}

'Bhumibol Adulyadej Hospital, Bangkok, Thailand (nootpiya@gmail.com) ${ }^{2}$ Thammasat University, Bangkok, Thailand

${ }^{3}$ Thammasat University Hospital, Khlong Nueng, Thailand

Background: Endometrial cancer (EC) is the 6th most frequent of cancer in the world. GLOBOCAN in year 2020 reported that 417,367 new cases of endometrial cancer worldwide. In Thailand, EC is the 6th most frequent cancer after breast, colon, cervix, liver, and lung cancers. There were 4,524 new cases of EC in Thai women in year 2020. Current treatment of EC is total hysterectomy with bilateral salpingo-oopherectomy. Bilateral pelvic lymphadenectomy, omentectomy, and paraaortic lymph node (LN) sampling were needed for surgical staging in EC. Adjuvant radiotherapy or concurrent chemoradiation (CCRT) was designed after corrected staging of EC. According to the International Federation of Gynecology and Obstetrics (FIGO) stage, histopathology, histological grading, depth of myometrial invasion (MI) and present of lymph-vascular invasion (LVSI) were the prognostic factors of EC. Previous studies had demonstrated that thrombocytosis was the poor prognostic factor of many cancer including ovary, cervix, vulvar, and breast. Thrombocytosis was also the prognostic factor in EC that affected disease-free survival (DFS) and overall survival (OS). In the present study, the association of pretreatment 